\title{
MONOTONICITY OF THE ZEROS OF A CROSS PRODUCT OF BESSEL FUNCTIONS
}

\author{
Lee Lorch
}

\begin{abstract}
The principal result here is that each positive zero of the function $J_{\nu+\beta}(x) / J_{\nu}(x)+\alpha^{\beta} I_{\nu+\beta}(\alpha x) / I_{\nu}(\alpha x)$ is an increasing function of $\nu$ in $-\beta / 2 \leq \nu<$ $\infty$ when $\alpha, \beta, k$ are fixed, $\alpha>0,0<\beta \leq 1, k=1,2,3, \ldots$ This implies that the $k$-th positive zero of $J_{\nu}(x) I_{\nu}^{\prime}(x)-I_{\nu}(x) \bar{J}_{\nu}^{\prime}(x)$ is an increasing function of $\nu,-\frac{1}{2} \leq$ $\nu<\infty, k=1,2, \ldots$, a result relevant to work of M. S. Ashbaugh and R. D. Benguria on eigenvalues in the clamped plate problem for the ball. The functions $J_{\nu}(x)$ and $I_{\nu}(x)$ are the Bessel and modified Bessel functions, respectively.
\end{abstract}

\section{Background and statement of main result}

Motivated by their appearance as eigenvalues in the clamped plate problem for the ball, M. S. Ashbaugh and R. D. Benguria (private communication) have conjectured that the positive zeros of

$$
f(x)=J_{\nu}(x) I_{\nu}^{\prime}(x)-J_{\nu}^{\prime}(x) I_{\nu}(x)
$$

increase with $\nu \geq-\frac{1}{2} . J_{\nu}(x)$ and $I_{\nu}(x)$ are the customary Bessel and modified Bessel functions [6]. Their conjecture will be verified here (Corollary 1), and also in a somewhat extended form (Theorem 1).

The proofs will employ, i.a., the recursion formulae $[6, \S 3.2(4)$, p. $45, \S 3.71(4)$, p. 79]

$$
x J_{\nu}^{\prime}(x)-\nu J_{\nu}(x)=-x J_{\nu+1}(x),
$$

and

$$
x I_{\nu}^{\prime}(x)-\nu I_{\nu}(x)=x I_{\nu+1}(x) .
$$

From them it follows immediately that the $k$-th positive zero $\gamma_{\nu k}$ of $f(x)$ is also the $k$-th positive zero of

$$
\varphi(x)=\frac{J_{\nu+1}(x)}{J_{\nu}(x)}+\frac{I_{\nu+1}(x)}{I_{\nu}(x)} .
$$

The denominators in (1.4) do not distort the discussion. If $x \neq 0$, then $I_{\nu}(x)>0$, $\nu>-1$ (as is evident from the power series [6, $\S 3.7(2)$, p. 77]); a positive zero of $J_{\nu}(x)$ cannot be a zero of $f(x)$ since $J_{\nu}(x)$ and $J_{\nu}^{\prime}(x)$ cannot vanish simultaneously, $x \neq 0$ $[6, \S 15.21$, p. 479$]$.

Received April 14, 1993, revised August 7, 1993.

1991 Mathematics Subject Classification. Primary: 33C10, 33B30, 34L15.

Key words and phrases. Bessel functions, zeros, monotonicity, eigenvalues.

This work received partial support from the Natural Sciences and Engineering Research Council of Canada. 
It is just as easy to consider the more general situation in which $\gamma(k, \nu, \alpha, \beta)$ denotes the $k$-th positive zero of an extension of $\varphi(x)$, namely

$$
\Phi(\alpha, \beta, \nu, x)=\Phi(\nu, x)=\frac{J_{\nu+\beta}(x)}{J_{\nu}(x)}+\alpha^{\beta} \frac{I_{\nu+\beta}(\alpha x)}{I_{\nu}(\alpha x)},
$$

so that $\gamma_{\nu k}=\gamma(k, \nu, 1,1)$.

The main result can now be formulated:

Theorem 1. Each zero $\gamma(k, \nu, \alpha, \beta)$ of (1.5) is an increasing function of $\nu,-\beta / 2 \leq$ $\nu<\infty$, for fixed $\alpha, \beta, k, \alpha>0,0<\beta \leq 1, k=1,2, \ldots$

When $\alpha=\beta=1$ this becomes the Ashbaugh-Benguria conjecture:

Corollary 1. The $k$-th positive zero $\gamma_{\nu k}$ of (1.1) and (1.4) is an increasing function of $\nu,-\frac{1}{2} \leq \nu<\infty$, for each fixed $k=1,2, \ldots$.

For the proof some results of [2] and [4] are required. As usual, $j_{\nu k}$ denotes the $k$-th positive zero of $J_{\nu}(x), j_{\nu k}^{\prime}$ the $k$-th positive zero of $J_{\nu}^{\prime}(x)$.

Clearly, $\Phi(\nu, x)>0,0<x<j_{\nu 1}$, since $j_{\nu+\beta, 1}>j_{\nu 1}, \beta>0[6, \S 15.6$, p. 508]. Also, $\Phi\left(\nu, j_{\nu 1}-\right)=+\infty$. Hence $\gamma(1, \nu, \alpha, \beta)>j_{\nu 1}$.

Theorem A ([2] Lemma 2.3). For each fixed $\beta(0<\beta \leq 1)$ and each $x>0(x \neq$ $\left.j_{\nu k}, k=1,2, \ldots\right)$, the function $J_{\nu+\beta}(x) / J_{\nu}(x)$ decreases as $\nu$ increases, $-(\beta+1) / 2 \leq$ $\nu<\infty, \nu>-1$.

Theorem B ([2] Lemma 2.5). For each fixed $\beta(0<\beta \leq 1)$ and each fixed $\nu \geq-\beta / 2$, the function $J_{\nu+\beta}(x) / J_{\nu}(x)$ increases with $x$ in each interval $j_{\nu k}<x<j_{\nu, k+1}, k=$ $0,1, \ldots$, where $j_{\nu 0}=0$.

Theorem C ([4] Theorem 1(8)). For each fixed $\beta>0, \nu>-1, \nu>-\beta / 2$, the positive function $I_{\nu+\beta}(x) / I_{\nu}(x)$ increases to 1 as $x$ increases, $0<x<\infty$.

\section{Auxiliary results}

For Theorem 1 to have any content it is necessary to know that $\gamma(k, \nu, \alpha, \beta)$ exists for the specified values of the parameters. This will be established in the next theorem which provides also bounds.

Theorem 2. For fixed $\alpha, \beta, \nu, k, \alpha>0,0<\beta \leq 1, k=1,2, \ldots, \nu \geq-\beta / 2$, the function $\gamma(k, \nu, \alpha, \beta)$ exists. Moreover, $j_{\nu k}<\gamma(k, \nu, \alpha, \beta)<j_{\nu+\beta, k}$.

Proof. Clearly, $\Phi(\nu, x)>0,0<x<j_{\nu 1}$, since $j_{\nu+\beta, 1}>j_{\nu 1}, \beta>0[6, \S 15.6$, p. 508]. Also, $\Phi\left(\nu, j_{\nu 1}-\right)=+\infty$. Hence $\gamma(1, \nu, \alpha, \beta)>j_{\nu 1}$. For each $k=1,2, \ldots$,

$$
\Phi\left(\nu, j_{\nu k}+\right)=-\infty, \Phi\left(\nu, j_{\nu+\beta, k}\right)=\alpha^{\beta} \frac{I_{\nu+\beta}\left(\alpha j_{\nu+\beta, k}\right)}{I_{\nu}\left(\alpha j_{\nu+\beta, k}\right)}>0
$$

so that a zero of $\Phi(\nu, x)$ exists in $j_{\nu k}<x<j_{\nu+\beta, k}$. Furthermore, this zero is unique, since $\Phi(\nu, x)$ is an increasing function in that interval according to Theorems B and C. The stated bounds are established as well.

Corollary 2. Let $\nu \geq-1 / 2$.

(i) For $k=1,2, \ldots$, the positive zeros $\gamma_{\nu k}$ of (1.1) and (1.4) satisfy the inequalities $j_{\nu k}<\gamma_{\nu k}<j_{\nu+1, k}$.

(ii) If $\nu \geq 0$, then also $\gamma_{\nu k}<j_{\nu, k+1}^{\prime}$. 
(iii) If $-1 / 2 \leq \nu<0$, then also $\gamma_{\nu k}<j_{\nu k}^{\prime}$.

Proof. (i) is evident, since $\gamma_{\nu k}=\gamma(k, \nu, 1,1)$.

(ii) The recursion formula (1.3) implies $I_{\nu}^{\prime}(x)>0$ when $\nu \geq 0$ Hence $J_{\nu}\left(\gamma_{\nu k}\right)$ and $J_{\nu}^{\prime}\left(\gamma_{\nu k}\right)$ must be of the same sign. It follows that $\gamma_{\nu k}<j_{\nu, k+1}^{\prime}$, as asserted, when $\nu \geq 0$.

(iii) This too follows from the common sign of $J_{\nu}\left(\gamma_{\nu k}\right)$ and $J_{\nu}^{\prime}\left(\gamma_{\nu k}\right)$, itself an obvious consequence of $I_{\nu}^{\prime}\left(\gamma_{\nu k}\right)>0$. The positivity of $I_{\nu}^{\prime}\left(\gamma_{\nu k}\right),-1 / 2 \leq \nu<0$, follows from the unimodality with respect to $\nu$ of $i_{\nu}^{\prime}$, the positive zero of $I_{\nu}^{\prime}(x)$, in $-1<\nu<0$. The unimodality is shown by C. G. Kokologiannaki, M. E. Muldoon and P. D. Siafarikas [3, $\S 4$, Remark]. Specifically, they establish (a) that there is a unique $i_{\nu}^{\prime}$ for $-1<\nu<0$, (b) that $i_{\nu}^{\prime}$ increases as $\nu$ increases from -1 to $\nu_{0}=-.569 \ldots$, (c) that $i_{\nu}^{\prime}$ decreases in the interval $\nu_{0}<\nu<0$, and (d) that $i_{\nu_{0}}^{\prime}=.778 \ldots$.

In particular, this implies that $I_{\nu}^{\prime}(x)>0$ for $x>i_{-1 / 2}^{\prime}$ when $-1 / 2<\nu<0$, since, for each $\nu$ in $-1<\nu<0, I_{\nu}\left(i_{\nu}^{\prime}\right)$ is the minimum of $I_{\nu}(x), 0<x<\infty$.

That the latter is the case follows from the differential equation satisfied by $y=$ $I_{\nu}(x)[6, \S 3.7(1)$, p. 77$]$

$$
x^{2} y^{\prime \prime}+x y^{\prime}-\left(x^{2}+\nu^{2}\right) y=0
$$

which shows that $I_{\nu}^{\prime \prime}(x)>0$ whenever $I_{\nu}^{\prime}(x)=0$.

Now, from (i) and the monotonicity of $j_{\nu 1}$,

$$
\gamma_{\nu k}>j_{\nu 1} \geq j_{-1 / 2,1}=\pi / 2>i_{-1 / 2}^{\prime}, \quad-\frac{1}{2} \leq \nu<0
$$

Hence the proof can be concluded as before.

Remarks. Theorem 4.1 of [3] actually discusses a function more general than $I_{\nu}(x)$. In the present notation, this function is

$$
H_{\nu}(x, \alpha)=\alpha I_{\nu}(x)+x I_{\nu}^{\prime}(x) .
$$

From the recursion formula (1.3), this can be written as

$$
H_{\nu}(x, \alpha)=(\alpha+\nu) I_{\nu}(x)+x I_{\nu+1}(x) .
$$

In [3] the existence and uniqueness of a positive zero of $H_{\nu}(x, \alpha),-1<\nu<0, \alpha+\nu<0$ is established.

A different approach can be employed which applies to the somewhat more general function

$$
H_{\nu}(x, \alpha, \beta)=(\alpha+\nu) I_{\nu}(x)+x I_{\nu+\beta}(x)
$$

Specifically:

$A$ necessary and sufficient condition that $H_{\nu}(x, \alpha, \beta)$ possess a positive zero when $\beta>0$ and $\nu>-1$ is that $\alpha+\nu<0$. The zero is unique.

Proof.

$$
\psi(x)=\frac{H_{\nu}(x, \alpha, \beta)}{I_{\nu}(x)}=\alpha+\nu+x \frac{I_{\nu+\beta}(x)}{I_{\nu}(x)}
$$

Then $\psi(0)=\alpha+\nu$, while from Theorem $\mathrm{C}, \psi(x)$ increases from this value to $+\infty$ as $x$ increases from 0 to $\infty$. The conclusion is now obvious. 


\section{An auxiliary result}

The proof of Theorem 1 will use also an analogue of [2, Lemma 2.1]:

Theorem 3. If $x>0$ and $\beta>0$ are fixed, then $I_{\nu+\beta}(x) / I_{\nu}(x)$ decreases as $\nu$ increases in $-(\beta+1) / 2 \leq \nu<\infty$, provided $\nu>-1$.

Proof. The conclusion is equivalent, for small $\varepsilon>0$, since $I_{\nu}(x)>0$, to

$$
\Delta:=I_{\nu+\beta}(x) I_{\nu+\varepsilon}(x)-I_{\nu+\beta+\varepsilon}(x) I_{\nu}(x)>0 .
$$

This follows from the formula $[6, \S 13.72(2)$, p. 441], valid for $\mu+\nu>-1$,

$$
I_{\mu}(x) I_{\nu}(x)=(2 / \pi) \int_{0}^{\pi / 2} I_{\mu+\nu}(x)(2 x \cos \theta) \cos \{(\mu-\nu) \theta\} d \theta
$$

From (3.2) it follows, for $\nu>-(\beta+1+\varepsilon) / 2$, that

$$
\Delta=(2 / \pi) \int_{0}^{\pi / 2} I_{2 \nu+\beta+\varepsilon}(2 x \cos \theta)[\cos \{(\beta-\varepsilon) \theta\}-\cos \{(\beta+\varepsilon) \theta\}] d \theta,
$$

and so

$$
\Delta=(4 / \pi) \int_{0}^{\pi / 2} I_{2 \nu+\beta+\varepsilon}(2 x \cos \theta) \sin (\beta \theta) \sin (\varepsilon \theta) d \theta
$$

This is clearly positive for $0<\varepsilon \leq 2$ and $0<\beta \leq 2$, again since $I_{\nu}(x)>0$.

It is not necessary to consider larger $\varepsilon$. To remove the restriction $\beta \leq 2$, it suffices, for any larger $\beta$, to decompose $I_{\nu+\beta}(x) / I_{\nu}(x)$ into an appropriate product of (decreasing) factors of the same form in which each factor has a numerator and a dominator whose orders do not differ by more than 2 .

Remark. In Theorem 3, the requirement that $\nu>-1$ (relevant when $\beta \geq 1$ ) cannot be dropped, since $I_{-n}(x)=I_{n}(x), n=1,2, \ldots[6, \S 3.71$ (8), p. 79].

\section{Proof of Theorem 1}

This proof is modelled after that of [2, Theorem 3.2]. Let $\gamma(k, \nu, \alpha, \beta)$ be abbreviated to $\gamma_{\nu}$, with $\alpha, \beta, k$ fixed.

From Theorem A [2, Lemma 2.3] and Theorem 3, it is evident that $\Phi(\nu, x)$, as defined by (1.5), decreases as $\nu$ increases, $-(\beta+1) / 2 \leq \nu<\infty$ for fixed $x>0$. Hence, for $\varepsilon>0$,

$$
\Phi\left(\nu+\varepsilon, \gamma_{\nu}\right)<\Phi\left(\nu, \gamma_{\nu}\right)=0, \quad-(\beta+1) / 2 \leq \nu<\infty, 0<\beta \leq 1,
$$

from the definition of $\gamma_{\nu}$.

From Theorem B [2, Lemma 2.5] and Theorem C [4,Theorem 1(8)], it is equally evident that $\Phi(\nu, x)$ is an increasing function of $x$ for fixed $\nu>-1,-\beta / 2 \leq \nu<$ $\infty, 0<\beta \leq 1, k=1,2, \ldots$. Hence the equation $\Phi\left(\nu+\varepsilon, \gamma_{\nu+\varepsilon}\right)=0$, valid by definition of $\gamma_{\nu+\varepsilon}$, implies $\gamma_{\nu+\varepsilon}>\gamma_{\nu}$, establishing the theorem. 


\section{The Turanian for $I_{\nu}(x)$}

Theorem 3, as expressed by (3.1), generalizes the positivity of the Turanian, $T_{\nu}(x)$, of $I_{\nu}(x)$, i.e.,

$$
T_{\nu}(x):=I_{\nu}^{2}(x)-I_{\nu-1}(x) I_{\nu+1}(x)>0, \quad \nu>-\frac{1}{2}, x>0 .
$$

Indeed, $T_{-1 / 2}(x)$ is also positive, $x>0$, as follows directly from the definitions

$$
\begin{aligned}
I_{-1 / 2}(x) & =\left(\frac{2}{\pi}\right)^{1 / 2} \frac{\cosh x}{x^{1 / 2}} \\
I_{1 / 2}(x) & =\left(\frac{2}{\pi}\right)^{1 / 2} \frac{\sinh x}{x^{1 / 2}} \\
I_{-3 / 2}(x) & =\left(\frac{2}{\pi}\right)^{1 / 2}\left[\frac{\sinh x}{x^{1 / 2}}-\frac{\cosh x}{x^{3 / 2}}\right] .
\end{aligned}
$$

Further, putting $\beta=\varepsilon$ in (3.1) and then replacing $\nu$ by $\nu-\varepsilon$ yields an inequality which generalizes (5.1), i.e., for $\varepsilon \neq 0$,

$$
T_{\nu, \varepsilon}(x):=I_{\nu}^{2}(x)-I_{\nu-\varepsilon}(x) I_{\nu+\varepsilon}(x)>0, \quad \nu>-\frac{1}{2}, x>0,
$$

as may be inferred from (3.3), which now takes the form

$$
T_{\nu, \varepsilon}(x)=(4 / \pi) \int_{0}^{\pi / 2} I_{2 \nu}(2 x \cos \theta) \sin ^{2} \varepsilon \theta d \theta, \quad \nu>-\frac{1}{2} .
$$

The inequalities (5.1) and (5.2) cannot be extended to all $\nu$. Even the more restricted (5.1) fails for $\nu=-3 / 2$, although it holds whenever $\nu$ is any integer (positive, negative or zero) since $I_{-n}(x)=I_{n}(x)[6, \S 3.71$ (8), p. 79].

An easy calculation establishes that

$$
T_{-3 / 2}(x)=\frac{2}{\pi x}\left[-1+\frac{\sinh x \cosh x}{x}-\frac{2 \cosh ^{2} x}{x^{2}}\right],
$$

an increasing function which is negative for $0<x<x_{0}$ and positive for $x_{0}<x<\infty$, where $x_{0}=2.2648858 \ldots$.

From (5.3) it is evident that

$$
\begin{gathered}
T_{\nu, \varepsilon}(x)<T_{\nu, \varepsilon+\eta}(x), 0<\varepsilon \leq 1, \eta>0, \nu>-\frac{1}{2}, x>0 \\
T_{\nu, \varepsilon}(x)<T_{\nu, \varepsilon}(x+\delta), \nu>-\frac{1}{2}, \varepsilon>0, \delta>0
\end{gathered}
$$

and

$$
T_{\nu, \varepsilon}(x)>T_{\nu+\mu, \varepsilon}(x), \nu>-\frac{1}{2} \varepsilon, \nu>-\frac{1}{2}, x>0, \varepsilon>0, \mu>0,
$$

i.e., that $T_{\nu, \varepsilon}(x)$ is an increasing function of $\varepsilon$ and $x$, but a decreasing function of $\nu$. 
Remarks. 1. Whether or not $T_{\nu, \varepsilon}(x)>0, x>0$, for $-1<\nu \leq-1 / 2$ as well as for the values $\nu>-1 / 2$ (for which positivity has been shown above) is an unanswered question.

2. Martin Muldoon, using Maple V, has kindly performed calculations which support the conjecture that $T_{\nu, \varepsilon}(x)>0, x>0,0<\varepsilon \leq 1$, also for $-1<\nu \leq-1 / 2$. In the course of preparation of this paper he called inequality (5.2) to my attention as well as the fact that it can be inferred from the representation (3.2). He noted that this extends an inequality established differently for the case $\nu \geq 0, \varepsilon=1$, of (5.2) i.e., the case $\nu \geq 0$ of (5.1), by D. E. Amos [1, p. 243]. In the same context, he observed that (5.6) could be established and that this generalizes a result due to E. Neuman [5, Theorem 6]. He pointed out that some other results of [5], when reformulated via appropriate recursion formulae, could be seen as direct consequences of [4]. I thank him for these observations and for his suggestion that they be incorporated herein.

3. For $-1 / 2 \leq \nu \leq 0$, the inequality (5.1) can be established without appealing to the representation (3.2). The recursion formula $[6, \S 3.71(1)$, p. 79$]$

$$
I_{\nu-1}(x)=I_{\nu+1}(x)+\frac{2 \nu}{x} I_{\nu}(x)
$$

permits rewriting $T_{\nu}(x)$ as

$$
T_{\nu}(x)=I_{\nu}^{2}(x)-I_{\nu+1}^{2}(x)-\frac{2 \nu}{x} I_{\nu}(x) I_{\nu+1}(x)
$$

This makes the inequality (5.1) obvious for $-1 / 2<\nu \leq 0$ from Theorem $\mathrm{C}[4$, Theorem 1 (8)]. For $\nu=-1 / 2,(5.1)$ follows directly from the definitions of $I_{\nu}(x)$ for $\nu=-1 / 2$ and $\nu=1 / 2$.

\section{References}

1. D. E. Amos, Computation of modified Bessel functions and their ratios, Math. Comp. 28 (1974), 239-251.

2. M. E. H. Ismail and M. E. Muldoon, Monotonocity of the zeros of a cross product of Bessel functions, SIAM J. Math. Anal. 9 (1978), 759-767.

3. C. G. Kokologiannaki, M. E. Muldoon and P. D. Siafarikas, A unimodal property of purely imaginary zeros of Bessel and related functions, Canadian Math. Bull. (to appear).

4. L. Lorch, Inequalities for some Whittaker functions, Arch. Math. (Brno) 3 (1967), 1-9.

5. E. Neuman, Inequalities involving modified Bessel functions of the first kind, J. Math. Anal. Appl. 171 (1992), 532-536.

6. G. N. Watson, A Treatise on the Theory of Bessel Functions, 2nd ed. The University Press, Cambridge 1960.

Department of Mathematics, York University, 4700 Keele Street, North York, Ontario, CANADA M3J 1 P3. 